\title{
One-loop determinant of Dirac operator in non-renormalizable models
}

\author{
A.A. Osipov, B. Hiller, A.H. Blin \\ Centro de Física Teórica, Departamento de Física da Universidade de Coimbra, 3004-516 Coimbra, Portugal \\ Received 16 December 1999; accepted 18 January 2000 \\ Editor: R. Gatto
}

\begin{abstract}
We use proper-time regularizations to define the one-loop fermion determinant in the form suggested by Gasser and Leutwyler some years ago. We show how to obtain the polynomial by which this definition of $\ln \operatorname{det} D$ needs to be modified in order to arrive at the fermion determinant whose modulus is invariant under chiral transformations. As an example it is shown how the fundamental symmetries associated with the NJL model are preserved in a consistent way. C 2000 Elsevier Science B.V. All rights reserved.
\end{abstract}

\section{Introduction}

In the path-integral formulation of quantum theory the effective action involves, after integration over the fermionic fields, the functional determinant of the differential Dirac operator $D$ in the presence of external sources. The central object in the calculation of the effective action is always the quantity $\ln \operatorname{det} D$. We start with the definition

$$
\begin{aligned}
\ln \operatorname{det} D= & -\frac{1}{2} \int_{0}^{\infty} \frac{d T}{T} \rho\left(T, \Lambda^{2}\right) \operatorname{Tr}\left(e^{-T \bar{D}^{2}}\right) \\
& -\int d^{4} x P(v, a, \sigma, \pi),
\end{aligned}
$$

which allows the Schwinger proper-time method to be applied to fermions, involving the square of the operator $\bar{D}$. Here $\bar{D} \equiv \gamma_{5} D$ has been introduced in [1], $D$ is the Dirac operator in the presence of external vector $\left(v_{\mu}\right)$, axial-vector $\left(a_{\mu}\right)$, scalar $(\sigma)$ and pseudoscalar $(\pi)$ sources. This definition of $\ln \operatorname{det} D$ allows to treat the real and imaginary parts of $\ln \operatorname{det} D$ on equal footing, as opposite to the $D^{\dagger} D$ definition. The polynomial $P(v, a, \sigma, \pi)$, which depends only on the external fields, is fixed by requiring the modulus of the fermion determinant to be invariant under chiral transformations. It has been worked out in the context of a renormalizable theory [1]. The present work represents an extension of the results of [1] to the case of non-renormalizable models and in particular to incorporate explicitly the process of dynamical chiral symmetry breaking of the Nambu-Jona-Lasinio (NJL) model [2]. As an alternative method one can use the integral representation of the complex power for the pseudo differential operator [3]. In the latter case an unambiguous definition of the determinant of the Dirac operator is obtained. The determinant is shown to be vector gauge invariant and to yield the correct axial and scale anomalies. 
We consider a class of regularization schemes (proper-time regularizations) which can be incorporated in this expression through the kernel $\rho\left(T, \Lambda^{2}\right)$. These regularizations allow to shift in loop momenta. A typical example is the proper-time cutoff where the kernel ${ }^{t} \rho\left(T, \Lambda^{2}\right)$ is equal to

${ }^{t} \rho\left(T, \Lambda^{2}\right)=\Theta\left(T-\frac{1}{\Lambda^{2}}\right)$.

Another choice for the kernel can be the covariant Pauli-Villars cutoff [4]

${ }^{c} \rho\left(T, \Lambda^{2}\right)=1-\left(1+T \Lambda^{2}\right) e^{-T \Lambda^{2}}$,

which leads to the well-known effective potential of the NJL model [2]. The result is ${ }^{1}$

$$
\begin{aligned}
V(m)= & \frac{m^{2}}{2 G}\left(1-\frac{N_{c} G \Lambda^{2}}{4 \pi^{2}}\right) \\
& +\frac{N_{c}}{8 \pi^{2}}\left[m^{4} \ln \left(1+\frac{\Lambda^{2}}{m^{2}}\right)\right. \\
& \left.-\Lambda^{4} \ln \left(1+\frac{m^{2}}{\Lambda^{2}}\right)\right] .
\end{aligned}
$$

Both of the kernels (2) and (3) have been used in many papers, for example, see papers [6,7] and [8] correspondingly. A wide set of possibilities for the kernel $\rho\left(T, \Lambda^{2}\right)$ have been considered in the papers $[9,10]$.

The counterterms $P(v, a, \sigma, \pi)$ in formula (1) can be fixed from the transformation properties of $\ln \operatorname{det} D$. We consider here the case of chiral gauge theories with the $S U(2)_{L} \times S U(2)_{R} \times U(1)_{V}$ chiral symmetry. Explicitly, let $D$ be equal to

$D=\gamma^{\mu}\left(i \partial_{\mu}+v_{\mu}+a_{\mu} \gamma_{5}\right)-\sigma+i \gamma_{5} \pi$,

where $v_{\mu}=v_{\mu}^{i} \tau_{i}, a_{\mu}=a_{\mu}^{i} \tau_{i}, \pi=\pi^{a} \tau_{a}, \sigma=\sigma^{a} \tau_{a}, \tau_{a}$ $=\left(1, \tau_{i}\right),\left[\tau_{i}, \tau_{j}\right]=2 i \epsilon_{i j k} \tau_{k}, \quad i=1,2,3$. The corre-

\footnotetext{
${ }^{1}$ See, for instance, [5] and references in it.
}

sponding chiral transformations of the external fields are given by

$$
\begin{aligned}
& \delta v_{\mu}=\partial_{\mu} \alpha+i\left[\alpha, v_{\mu}\right]+i\left[\beta, a_{\mu}\right], \\
& \delta a_{\mu}=\partial_{\mu} \beta+i\left[\alpha, a_{\mu}\right]+i\left[\beta, v_{\mu}\right], \\
& \delta \sigma=i[\alpha, \sigma]-\{\beta, \pi\}, \\
& \delta \pi=i[\alpha, \pi]+\{\beta, \sigma\} .
\end{aligned}
$$

Here $\alpha=\alpha_{i} \tau_{i}$ is the infinitesimal transformation generated by the vector currents and $\beta=\beta_{i} \tau_{i}$ is a chiral transformation. The transformation law of $\ln \operatorname{det} D$ in this case is known explicitly [11]:

$\delta \ln \operatorname{det} D=\frac{i N_{c}}{(4 \pi)^{2}} \int d^{4} x \operatorname{Tr}_{f}(\beta \Omega)$,

where

$$
\begin{aligned}
\Omega= & \varepsilon^{\alpha \beta \mu \nu}\left[v_{\alpha \beta} v_{\mu \nu}+\frac{4}{3} \nabla_{\alpha} a_{\beta} \nabla_{\mu} a_{\nu}+\frac{2 i}{3}\left\{v_{\alpha \beta}, a_{\mu} a_{\nu}\right\}\right. \\
& \left.+\frac{8 i}{3} a_{\mu} v_{\alpha \beta} a_{\nu}+\frac{4}{3} a_{\alpha} a_{\beta} a_{\mu} a_{\nu}\right] .
\end{aligned}
$$

The field strength tensor $v_{\mu \nu}$ associated with $v_{\mu}$ is defined as

$v_{\mu \nu}=\partial_{\mu} v_{\nu}-\partial_{\nu} v_{\mu}-i\left[v_{\mu}, v_{\nu}\right]$

and $\nabla_{\mu} a_{\nu}$ stands for

$\nabla_{\mu} a_{\nu}=\partial_{\mu} a_{\nu}-i\left[v_{\mu}, a_{\nu}\right]$.

Our aim now is to calculate the polynomial $P(v, a, \sigma, \pi)$ in the framework of a nonrenormalizable approach. Let us note that $P(v, a, \sigma, \pi)$ is unique up to a chirally invariant polynomial. One can always choose $P$ in such a manner that the determinant is not modified if the external fields $a_{\mu}$ and $\pi$ are switched off. In paper [1] it has been shown how to do this for renormalizable theories. There are two essential differences in our case. The first one is that we have to use a regularization with finite cutoff $\Lambda$. The $\zeta$-function technique used in [1] is not good for that because it does not lead to the correct description of the spontaneous chiral symmetry breaking phenomena. The second one is also related to the cutoff dependence of the result. As we shall show, the polynomial $P(v, a, \sigma, \pi)$ gets now systematically contributions from the terms which would vanish in the limit $\Lambda \rightarrow \infty$. This fact renders its evaluation rather technical. 


\section{Counterterms and symmetry}

To illustrate our consideration we shall discuss the NJL model with the $S U(2)_{L} \times S U(2)_{R}$ chiral symmetry. We use the model version with only the scalar-scalar and pseudoscalar-pseudoscalar type of four quark interactions. Integrating out the quark fields one obtains the action of the model in terms of scalar $\sigma \times 1$ and pseudoscalar $\pi=\pi_{i} \tau^{i}$ collective mesonic degrees of freedom

$S_{\text {coll }}=-i \ln \operatorname{det} D-\int d^{4} x \frac{(\sigma+m)^{2}+\pi^{2}}{2 G}$.

The Dirac operator $D$ is given by

$D=i \gamma^{\mu} \partial_{\mu}-m-\sigma+i \gamma_{5} \pi$,

where $m$ denotes the constituent quark mass generated in the process of spontaneous chiral symmetry breaking. Now in order to be able to derive the polynomial $P(\sigma, \pi)$, it is crucial to perform the symmetry transformations in the broken phase. In the phase with broken chiral symmetry the transformations (8) and (9) become

$\bar{\delta} \sigma=-\{\beta, \pi\}$,

$\bar{\delta} \pi=i[\alpha, \pi]+\{\beta, \sigma+m\}$

for the considered isospin content of scalar and pseudoscalar. Note that if one would first derive $P(\sigma, \pi)$ in the symmetric phase and then perform the shift $\sigma \rightarrow(\sigma+m)$, it would be necessary to calculate all orders of the proper-time expansion. All of them would contribute as a factor with a certain power $m$ to a fixed order in the fields. Therefore by constructing the symmetry transformations in the broken phase (16), (17), one achieves a resummation of an infinite number of terms of the symmetric phase.

Under global chiral transformations the change in the Dirac operator $\bar{D}=\gamma_{5} D$ is given by

$i \bar{\delta} \bar{D}=[\bar{D}, \alpha]+\left\{\bar{D}, \beta \gamma_{5}\right\}$.

Therefore, to get the related polynomial $P(\sigma, \pi)$ for this case one has to integrate the equality

$\bar{\delta} \ln \operatorname{det} D=0$, where $\ln \operatorname{det} D$ is defined according to Eq. (1). The variation of $P(\sigma, \pi)$ has to cancel the symmetry breaking part coming from the proper-time integral. In this way one gets

$\bar{\delta} P(\sigma, \pi)=\frac{-i}{8 \pi^{2}} \sum_{n=0}^{\infty} R_{n} \operatorname{tr}\left(\beta \gamma_{5} a_{n+1}\right)$,

where tr represents trace in internal space. In the case under consideration it includes summations over flavour, colour and Lorentz indexes: $\operatorname{tr}=\operatorname{tr}_{f} \operatorname{tr}_{c} \operatorname{tr}_{L}$. One can see that $P(\sigma, \pi)$ is not invariant under chiral transformations, picking up the contribution which is linear in $\beta$. The functions $R_{n}$ represent the integrals which appear in the result of the asymptotic expansion of the heat kernel

$$
\begin{aligned}
R_{n} & =-\int_{0}^{\infty} \rho\left(T, \Lambda^{2}\right) d\left[T^{n-1} e^{-T m^{2}}\right] \\
& =\int_{0}^{\infty} d T T^{n-2}\left[m^{2} T-(n-1)\right] e^{-T m^{2}} \rho\left(T, \Lambda^{2}\right) .
\end{aligned}
$$

These integrals yield the following expression for $R_{n}$ ${ }^{c} R_{n}=\frac{n ! \Lambda^{4}}{\left(\Lambda^{2}+m^{2}\right)^{n+1}}$.

This result corresponds to the kernel (3). For the case of $\rho\left(T, \Lambda^{2}\right)$ being equal to (2) one gets

${ }^{t} R_{n}=\left(\Lambda^{2}\right)^{1-n} \exp \left(-\frac{m^{2}}{\Lambda^{2}}\right)$.

In renormalizable theory the terms $R_{n}$ with $n \geq 2$ vanish in the limit $\Lambda \rightarrow \infty$. The same is also true if one applies the $\zeta$-function regularization. This property of renormalizable models extremely simplifies the problem. In non-renormalizable models all of $R_{n}$ terms contribute to the result.

The coefficients $a_{n} \equiv a_{n}(x, x)$ are the coincidence limit of the Seeley - DeWitt coefficients [9]. For our illustration we shall need the first four of them

$$
\begin{aligned}
a_{0}= & 1, \quad a_{1}=-Q, \quad a_{2}=\frac{1}{2} Q^{2}+\frac{1}{6} Q_{\mu \mu}+\frac{1}{12} F^{2} \\
a_{3}= & -\frac{1}{6} Q^{3}-\frac{1}{12}\left(\left\{Q, Q_{\mu \mu}\right\}+Q_{\mu}^{2}\right)-\frac{1}{60} Q_{\mu \mu \nu \nu} \\
& +\frac{1}{60}\left[F_{\mu \alpha ; \alpha}, Q_{\mu}\right]-\frac{1}{60}\left(2\left\{F^{2}, Q\right\}+F_{\mu \nu} Q F_{\mu \nu}\right) \\
& -\frac{1}{45} F_{\mu \alpha ; \alpha} F_{\mu \beta ; \beta}-\frac{1}{180} F_{\mu \alpha ; \beta} F_{\mu \alpha ; \beta} \\
& -\frac{1}{60}\left\{F_{\mu \nu}, F_{\mu \nu ; \alpha \alpha}\right\}+\frac{1}{30} F^{3} .
\end{aligned}
$$


Some comments are in order here. First, we deal in this case with the linear realization of chiral symmetry. It means that we have for $\bar{D}^{2}$ the following representation

$\bar{D}^{2}=\nabla_{\mu} \nabla^{\mu}+m^{2}+Q$,

where

$$
\begin{aligned}
\nabla_{\mu}= & \partial_{\mu}+A_{\mu}, \quad A_{\mu}=\gamma_{\mu} \gamma_{5} \pi, \\
Q= & \left(\sigma^{2}+2 m \sigma\right)+i \gamma^{\mu} \partial_{\mu} \sigma \\
& -2(m+\sigma) i \gamma_{5} \pi+3 \pi^{2} .
\end{aligned}
$$

Second, we wrote the coefficients (24) directly in Minkowski space. In this way one should understand summations over repeated Lorentz indexes to be implicit. We have used the following designations

$F_{\mu \nu}=\left[\nabla_{\mu}, \nabla_{\nu}\right]=\gamma_{\nu} \gamma_{5} \partial_{\mu} \pi-\gamma_{\mu} \gamma_{5} \partial_{\nu} \pi+\left[\gamma_{\nu}, \gamma_{\mu}\right] \pi^{2}$,

$F^{2}=F_{\mu \nu} F^{\mu \nu}, \quad F^{3}=F_{\mu \nu} F^{\nu \sigma} F_{\sigma \mu}$,

$Q_{\mu}=\left[\nabla_{\mu}, Q\right], \quad F_{\mu \nu ; \nu}=\left[\nabla_{\nu}, F_{\mu \nu}\right]$.

One has to calculate traces $\operatorname{tr}\left(\beta \gamma_{5} a_{n}\right)$ and integrate Eq. (20). The first three non-zero contributions are given by

$$
\begin{aligned}
& \operatorname{tr}\left(\beta \gamma_{5} a_{1}\right)=4 i N_{c} \bar{\delta} \boldsymbol{\pi}^{2}, \\
& \operatorname{tr}\left(\beta \gamma_{5} a_{2}\right) \\
& \quad=4 i N_{c} \bar{\delta}\left[\frac{1}{6}\left(\partial_{\mu} \boldsymbol{\pi}\right)^{2}-2 m \sigma \boldsymbol{\pi}^{2}-\sigma^{2} \boldsymbol{\pi}^{2}-\frac{2}{3} \boldsymbol{\pi}^{4}\right],
\end{aligned}
$$

$$
\begin{aligned}
\operatorname{tr}\left(\beta \gamma_{5} a_{3}\right)= & 4 i N_{c} \bar{\delta}\left[\frac{1}{60}\left(\partial_{\mu}^{2} \boldsymbol{\pi}\right)^{2}-\frac{1}{2} \boldsymbol{\pi}^{2}\left(\partial_{\mu} \sigma\right)^{2}\right. \\
& -\frac{1}{5} \boldsymbol{\pi}^{2}\left(\partial_{\mu} \boldsymbol{\pi}\right)^{2}-\frac{7}{60}\left(\partial_{\mu} \boldsymbol{\pi}^{2}\right)^{2} \\
& -\frac{1}{3}(\sigma+m) \partial_{\mu} \sigma \partial_{\mu} \boldsymbol{\pi}^{2}+\frac{1}{10} \boldsymbol{\pi}^{6} \\
& +\frac{1}{6}\left(\sigma^{2}+2 m \sigma\right)\left[3 \boldsymbol{\pi}^{2}\left(\sigma^{2}+2 m \sigma\right)\right. \\
& \left.+2 \boldsymbol{\pi}^{4}-\left(\partial_{\mu} \boldsymbol{\pi}\right)^{2}\right]-\frac{1}{3} m^{2} \boldsymbol{\pi}^{4} \\
& \left.-2 m \sigma \boldsymbol{\pi}^{2}-\sigma^{2} \boldsymbol{\pi}^{2}-\frac{2}{3} \boldsymbol{\pi}^{4}\right]
\end{aligned}
$$

Let us note that the last four terms from $a_{3}$ (see Eq. (24)) do not contribute to $\operatorname{tr}\left(\beta \gamma_{5} a_{3}\right)$.
On the other side, the first term in formula (1) contributes to the Lagrangian of collective fields as

$\mathscr{L}_{\text {coll }}^{(1)}=-\frac{1}{32 \pi^{2}} \sum_{n=0}^{\infty} J_{n} \operatorname{tr}\left(a_{n+1}\right)$,

where

$J_{n}=\int_{0}^{\infty} \frac{d T}{T^{2-n}} e^{-T m^{2}} \rho\left(T, \Lambda^{2}\right), \quad n=0,1,2, \ldots$.

We have from (34)

$$
\begin{aligned}
\mathscr{L}_{\text {coll }}^{(1)}= & \frac{N_{c}}{(2 \pi)^{2}}\left\{\left(\sigma^{2}+2 m \sigma+3 \pi^{2}\right) J_{0}\right. \\
& +\frac{1}{2}\left[\left(\partial_{\mu} \sigma\right)^{2}+\left(\partial_{\mu} \pi\right)^{2}\right. \\
& \left.\left.+4 m^{2} \pi^{2}-\left(\sigma^{2}+2 m \sigma+\pi^{2}\right)^{2}\right] J_{1}+\ldots\right\} .
\end{aligned}
$$

Using the identity

$m^{2} J_{n}+(2-n) J_{n-1}=R_{n-1}, \quad n=1,2,3, \ldots$

one can see, for instance, how symmetry breaking terms proportional to $\boldsymbol{\pi}^{2}$ are compensated in this expression by the contribution from (31). A fully chiral symmetric Lagrangian is therefore obtained at each order of the proper time expansion.

\section{Conclusions}

We used the one-loop fermion determinant in the form suggested by Gasser and Leutwyler some years ago [1] to extend it to be applicable to non-renormalizable models. In this way the real and imaginary parts of $\ln \operatorname{det} D$ can be calculated with the same input. One obtains the correct description of the chiral anomaly when regularization is switched off. However it is necessary to correct the real part of $\ln \operatorname{det} D$ by the polynomial $P(v, a, \sigma, \pi)$ to get the chiral invariant result for this case. We have shown how to get the chiral symmetry restoring polynomial $P(v, a, \sigma, \pi)$. The simplest way to do this is to calculate in the phase with broken chiral symmetry, rewriting the symmetry transformations especially for this case. The result is an extension of the form 
presented in the paper [1], to incorporate explicitly the process of dynamical chiral symmetry breaking of the NJL model.

For simplicity we have considered the NJL model without vector and axial-vector degrees of freedom. However, the result can be easily extended to the more general case.

\section{Acknowledgements}

This work is supported by grants provided by Fundação para a Ciência e a Tecnologia, PRAXIS XXI/BCC /7301/96, PRAXIS /C / FIS / 12247/ 1998, PESO/P/PRO/15127/1999 and NATO “'Outreach' Cooperation Program.

\section{References}

[1] J. Gasser, H. Leutwyler, Ann. Phys. (NY) 158 (1984) 142.

[2] Y. Nambu, G. Jona-Lasinio, Phys. Rev. 122 (1961) 345; 124 (1961) 246

[3] L.L. Salcedo, R.E. Arriola, Ann. Phys. (NY) 250 (1996) 1.

[4] W. Pauli, F. Villars, Rev. Mod. Phys. 21 (1949) 434.

[5] S. Ying, Ann. Phys. 250 (1996) 69.

[6] J. Bijnens, Phys. Rep. 265 (1996) 369.

[7] E.N. Nikolov, W. Broniowski, C.V. Christov, G. Ripka, K. Goeke, Nucl. Phys. A 608 (1996) 411.

[8] V. Bernard, A.A. Osipov, and Meißner, U.-G., Phys. Lett. B 285 (1992) 119; V. Bernard, A.H. Blin, B. Hiller, Y.P. Ivanov, A.A. Osipov, U.-G. Meißner, Ann. Phys. (NY) 249 (1996) 499.

[9] R.D. Ball, Phys. Rep. 182 (1989) 1.

[10] F. Döring, A. Blotz, C. Schüren, Th. Meissner, E. RuizArriola, K. Goeke, Nucl. Phys. A 536 (1992) 548.

[11] W.A. Bardeen, Phys. Rev. 184 (1969) 1848. 